\title{
Erratum to: Diagnostic criteria for slowly progressive insulin- dependent (type 1) diabetes mellitus (SPIDDM) (2012): report by the Committee on Slowly Progressive Insulin-Dependent (Type 1) Diabetes Mellitus of the Japan Diabetes Society
}

\author{
Shoichiro Tanaka' ${ }^{1}$ Masayuki Ohmori ${ }^{1} \cdot$ Takuya Awata $^{2} \cdot$ Akira Shimada $^{3} \cdot$ Satoshi Murao $^{4}$. \\ Taro Maruyama $^{5} \cdot$ Kyuzi Kamoi $^{6} \cdot$ Eiji Kawasaki $^{7} \cdot$ Koji Nakanishi $^{8} \cdot$ Masao Nagata $^{9} \cdot$ Sumie Fujii $^{10}$. \\ Hiroshi Ikegami $^{11}$ - Akihisa Imagawa ${ }^{12} \cdot$ Yasuko Uchigata $^{13} \cdot$ Minoru Okubo $^{14} \cdot$ Haruhiko Osawa $^{15}$. \\ Hiroshi Kajio $^{16}$ - Akio Kawaguchi ${ }^{1}$ Yumiko Kawabata ${ }^{11} \cdot$ Jo Satoh $^{17}$ • Ikki Shimizu ${ }^{18}$ Kazuma Takahashi $^{17}$. \\ Hideichi Makino $^{19} \cdot$ Hiromi Iwahashi $^{12} \cdot$ Junnosuke Miura $^{13} \cdot$ Kazuki Yasuda $^{20} \cdot$ Toshiaki Hanafusa $^{21}$. $^{2}$ \\ Tetsuro Kobayashi ${ }^{1,22} \cdot$ Committee on Type 1 Diabetes
}

Published online: 25 March 2015

(C) The Japan Diabetes Society 2015

\section{Erratum to: Diabetol Int DOI 10.1007/s13340-014-0199-2}

In the published report, the following information was inadvertently omitted:

In 2012, the Japan Diabetes Society established the Committee on Slowly Progressive Insulin-Dependent (Type 1) Diabetes Mellitus of the Japan Diabetes Society, which

The online version of the original article can be found under doi:10.1007/s13340-014-0199-2.

Tetsuro Kobayashi

tetsurou@yamanashi.ac.jp

1 Third Department of Internal Medicine, Interdisciplinary Graduate School of Medicine and Engineering, University of Yamanashi, 1110 Shimokato, Chuo, Yamanashi 409-3898, Japan

2 Department of Endocrinology and Diabetes, Saitama Medical University, Saitama, Japan

3 Department of Internal Medicine, Saiseikai Central Hospital, Tokyo, Japan

4 Department of Diabetes and Endocrinology, Takamatsu Hospital, Kagawa, Japan

5 Department of Internal Medicine, Saitama Social Insurance Hospital, Saitama, Japan

6 The Diabetes and Endocrine and Metabolism Disease Center, Ojiya General Hospital, Niigata, Japan

7 Department of Metabolism/Diabetes and Clinical Nutrition, Nagasaki University Hospital, Nagasaki, Japan

8 Department of Diabetes, Minami-isshiki Central Clinic, Shizuoka, Japan published a committee report in $J$ Japan Diab Soc 2013;56:590-597 (in Japanese). This is the English version of that report. Tetsuro Kobayashi, Chairman; Shoichiro Tanaka, Secretariat.

There were errors in the names of two authors:

The twelfth author should be shown as Hiroshi Ikegami. The seventeenth author should be shown as Hiroshi Kajio.
9 Department of Internal Medicine, Kakogawa West City Hospital, Hyogo, Japan

10 Diabetology and Endocrinology, Ishikawa Prefectural Central Hospital, Ishikawa, Japan

11 Department of Endocrinology, Metabolism and Diabetes, Kinki University School of Medicine, Osaka, Japan

12 Department of Metabolic Medicine, Graduate School of Medicine, Osaka University, Osaka, Japan

13 Diabetes Center, Tokyo Women's Medical University School of Medicine, Tokyo, Japan

14 Department of Endocrinology and Metabolism, Toranomon Hospital, Tokyo, Japan

15 Department of Molecular and Genetic Medicine, Ehime University Graduate School of Medicine, Ehime, Japan

16 Division of Diabetes, Metabolism and Endocrinology, Department of Internal Medicine, National Center for Global Health and Medicine Hospital, Tokyo, Japan

17 Department of Diabetes and Metabolism, Iwate Medical University, Iwate, Japan 
18 Department of Diabetic Medicine, The Sakakibara Heart Institute of Okayama, Okayama, Japan

19 Diabetes Center, Shiraishi Hospital, Ehime, Japan

20 Department of Metabolic Disorder, Diabetes Research

Center, Research Institute, National Center for Global Health and Medicine, Tokyo, Japan

21 Department of Internal Medicine (I), Osaka Medical College, Osaka, Japan

22 Okinaka Memorial Institute for Medical Research, Tokyo, Japan 